\title{
Nucleon-nucleon interactions via Lattice QCD: Methodology
}

\section{HAL QCD approach to extract hadronic interactions in lattice QCD}

\begin{abstract}
Sinya Aoki ${ }^{\mathrm{a}}$
Graduate School of Pure and Applied Sciences, University of Tsukuba, Ten-oh-dai 1-1-1, Tsukuba, Ibaraki 305-8571, Japan

Received: date / Revised version: date

Abstract. We review the potential method in lattice QCD, which has recently been proposed to extract nucleon-nucleon interactions via numerical simulations. We focus on the methodology of this approach by emphasizing the strategy of the potential method, the theoretical foundation behind it, and special numerical techniques. We compare the potential method with the standard finite volume method in lattice $\mathrm{QCD}$, in order to make pros and cons of the approach clear. We also present several numerical results for the nucleon-nucleon potentials.
\end{abstract}

PACS. 12.38.Gc Lattuce QCD calculations - 13.75.Cs Nucleon-nucleon interactions

\section{Introduction}

Thank to both a steady growth of computational powers and various innovations of numerical algorithms, we are now able to calculate static properties of light hadrons such as masses and decay constants at the physical point in the continuum limit of lattice QCD. See, for example, Ref. 1 for a recent review. One of the next targets in lattice QCD calculations is to extract hadronic interactions such as the scattering between stable hadrons, masses and widths of unstable particles and binding energies of multihadron states. A standard framework to evaluate scattering phase shifts in lattice QCD is the Lüshcer finite volume method 2, which relates the energy spectrum for two hadrons in a finite box with the elastic-scattering phase shift of two hadrons in the infinite volume. The method has been applied to various two-hadrons systems 3 .

An alternative but closely related approach to hadronic interactions in lattice QCD has recently been proposed and applied to the nucleon-nucleon $(N N)$ system $4,5,6$, In the method, one first calculate the $N N$ potential, and then extract physical observables such as the scattering phase shift by solving the Schrödinger equation with the potential obtained. The method has been widely applied to general hadronic interactions for baryon-baryon [7, 8, , 10,11, meson-baryon [12,13,14 and three nucleon [15, 16. systems, mainly by the HAL QCD (Hadron to Atomic nuclei from Lattice QCD) collaboration. See Refs. 17, 18, for reviews on recent activities.

In this paper, we review this potential method, called the HAL QCD method from the name of the collaboration,

a Address after April 1, 2013: Yukawa Institute for Theoretical Physics, Kyoto University, Kitashirakawa Oiwakecho, Sakyo-ku, Kyoto 606-8502, Japan focusing on the methodology of the approach such as the theoretical foundation and the numerical techniques.

\section{Strategy: HAL QCD method}

In this section we explain the strategy of the HAL QCD method, taking the $N N$ system as an explicit example.

\subsection{Lippmann-Schwinger equation}

A concept of the potential in quantum field theories may appear in the Lippmann-Scwhinger equation[19],

$$
|\alpha\rangle_{\text {in }}=|\alpha\rangle_{0}+\int d \beta \frac{|\beta\rangle_{0} T_{\beta \alpha}}{E_{\alpha}-E_{\beta}+i \epsilon}
$$

where the asymptotic in-state $|\alpha\rangle_{\text {in }}$ satisfies

$$
\left(H_{0}+V\right)|\alpha\rangle_{\text {in }}=E_{\alpha}|\alpha\rangle_{\text {in }},
$$

while the non-interacting state $|\alpha\rangle_{0}$ does

$$
H_{0}|\alpha\rangle_{0}=E_{\alpha}|\alpha\rangle_{0} .
$$

The off-shell $T$-matrix element $T_{\beta \alpha}$ is defined through the "potential" $V$ as

$$
T_{\beta \alpha}={ }_{0}\langle\beta|V| \alpha\rangle_{\text {in }} .
$$

This quantity is related to the on-shell $S$-matrix, $S=$ $1-i T$, as

$$
{ }_{0}\langle\beta|T| \alpha\rangle_{0}=2 \pi \delta\left(E_{\alpha}-E_{\beta}\right) T_{\beta \alpha} .
$$

So our task is to extract $T_{\beta \alpha}$ in lattice QCD simulations. 


\subsection{Nambu-Bethe-Salpeter wave functions}

The basic quantity in the HAL QCD method is the equaltime Nambu-Bethe-Salpeter(NBS) wave function 20, defined for the $N N$ system as

$$
\begin{aligned}
\Psi_{\alpha \beta, f g}^{\boldsymbol{k}, s_{1} s_{2}}(\boldsymbol{x}) & =\langle 0| T\left\{N_{\alpha, f}(\boldsymbol{r}, 0) N_{\beta, g}(\boldsymbol{r}+\boldsymbol{x}, 0)\right\} \\
& \times\left|N N, \boldsymbol{k}, s_{1} s_{2}\right\rangle_{\mathrm{in}},
\end{aligned}
$$

where $\langle 0|$ is the QCD vacuum state, $T$ represents the timeordered product, $\left|N N, \boldsymbol{k}, s_{1} s_{2}\right\rangle_{\text {in }}$ is the two-nucleon instate which has helicity $s_{1}, s_{2}$, the relative momentum $\boldsymbol{k}$ and the total energy $W_{\boldsymbol{k}}=2 \sqrt{\boldsymbol{k}^{2}+m_{N}^{2}}$ with the nucleon mass $m_{N}$ in the center of mass system. For the interpolating operator for nucleon, we take the local one given by $N_{\alpha, f}(x)=\epsilon_{a b c}\left(u^{a}(x)^{T} C \gamma_{5} d^{b}(x)\right) q_{\alpha . f}^{c}(x)$ with $x=(\boldsymbol{x}, t)$, where $a, b, c$ are color indices, $\alpha, f$ are spinor and flavor indices, $C=\gamma_{2} \gamma_{4}$ is the charge conjugation matrix, $q(x)=(u(x), d(x))$, and $u, d$ represent up and down quark fields, respectively. As we will see, a potential in the HAL QCD scheme is defined through the NBS wave function, so that it depends on the choice of $N_{\alpha, f}(x)$.

It is important to note that, as long as the total energy $W_{\boldsymbol{k}}$ is below the pion production threshold such that $W_{\boldsymbol{k}}<W_{\mathrm{th}} \equiv 2 m_{N}+m_{\pi}$ with the pion mass $m_{\pi}$, the NBS wave function at large $r \equiv|\boldsymbol{x}|$ satisfies the Helmholtz equation as

$$
\left[k^{2}+\nabla^{2}\right] \Psi_{\Gamma}^{A}(\boldsymbol{x}) \simeq 0, \quad k=|\boldsymbol{k}|,
$$

where we write $\Gamma=\alpha \beta, f g$ and $A=\boldsymbol{k}, s_{1} s_{2}$ for simplicity. Furthermore, the radial part of the NBS wave function for a given orbital angular momentum $L$, the total spin $S$ and the total isospin $I$ for the large $r$ is given by 21,6 ]

$$
\Psi^{A}(r ; L S I) \propto \frac{\sin \left(k r-L \pi / 2+\delta_{L S I}(k)\right)}{k r} e^{i \delta_{L S I}(k)},
$$

where $\delta_{L S I}(k)$ is the $N N$ scattering phase shift below the inelastic threshold, which appears in the $S$-matrix by the unitarity constraint. In the appendix, we derive eq. (8) for the scalar field, for simplicity, using the LippmannSchwinger equation.

\subsection{Non-local potential}

Our task now becomes to extract the scattering phase shift encoded in the NBS wave function. For this purpose, we define a non-local potential from the NBS wave function through the equation $4,5,6$,

$$
\left[E_{k}-H_{0}\right] \Psi_{\Gamma}^{A}(\boldsymbol{x})=\sum_{\Gamma_{a}} \int d^{3} y U_{\Gamma, \Gamma_{a}}(\boldsymbol{x}, \boldsymbol{y}) \Psi_{\Gamma_{a}}^{A}(\boldsymbol{y})
$$

where $E_{k}=k^{2} /(2 \mu)$ with the reduced mass $\mu=m_{N} / 2$, and $H_{0}=-\nabla^{2} /(2 \mu)$. First of all, the non-local potential $U(\boldsymbol{x}, \boldsymbol{y})$ is expected to be finite-ranged since massless particle exchanges between two nucleons are absent. Secondly, the potential is finite and renormalization scheme independent, since the NBS wave function $\Psi_{\Gamma}^{A}(\boldsymbol{x})$ is multiplicatively renormalized in QCD and the same renormalization factor appears in both sides of eq. (9). Thirdly, while Lorentz covariance is lost by taking the equal-time to define the NBS wave function in eq. (6) and the potential is defined through the non-relativistic Schrödinger equation (9), non-relativistic "approximation" has never been introduced to define $U(\boldsymbol{x}, \boldsymbol{y})$.

One of the most important properties for $U(\boldsymbol{x}, \boldsymbol{y})$ is that $U(\boldsymbol{x}, \boldsymbol{y})$ does not depend on energy (or more precisely momentum $\boldsymbol{k}$ ) or helicities $s_{1}, s_{2}$ of the particular NBS wave function. This can be shown by directly constructing such a non-local potential $U(\boldsymbol{x}, \boldsymbol{y})$ as 6 .

$U_{\Gamma_{a}, \Gamma_{b}}(\boldsymbol{x}, \boldsymbol{y})=\sum_{A_{a}, A_{b}}^{\left|\boldsymbol{k}_{a, b}\right|<k_{\mathrm{th}}}\left[E_{k_{a}}-H_{0}\right] \Psi_{\Gamma_{a}}^{A_{a}}(\boldsymbol{x}) \mathcal{N}_{A_{a}, A_{b}}^{-1} \Psi_{\Gamma_{b}}^{A_{b}}(\boldsymbol{y})$,

where $A_{a}=\boldsymbol{k}_{a}, s_{1}^{a} s_{2}^{a}$ etc. and $k_{\text {th }}$ is the threshold momentum which satisfies $W_{\mathrm{th}}=2 \sqrt{k_{\mathrm{th}}^{2}+m_{N}^{2}}$, so that the summations over $\boldsymbol{k}_{a}$ and $\boldsymbol{k}_{b}$ are restricted below the inelastic threshold. Here $\mathcal{N}^{-1}$ is the inverse of $\mathcal{N}$ defined from the inner product of the NBS wave functions as

$$
\mathcal{N}^{A_{a}, A_{b}}=\left(\Psi^{A_{a}}, \Psi^{A_{b}}\right) \equiv \sum_{\Gamma} \int d^{3} x \Psi_{\Gamma}^{A_{a}}(\boldsymbol{x})^{\dagger} \Psi_{\Gamma}^{A_{b}}(\boldsymbol{x}),
$$

and therefore the inverse satisfies

$$
\sum_{A_{c}}^{\left|\boldsymbol{k}_{c}\right|<k_{\mathrm{th}}} \mathcal{N}_{A_{b}, A_{c}}^{-1} \mathcal{N}^{A_{c}, A_{b}}=\delta_{A_{a}}^{A_{b}} \equiv \delta^{(3)}\left(\boldsymbol{k}_{a}-\boldsymbol{k}_{b}\right) \delta_{s_{1}^{a}}^{s_{1}^{b}} \delta_{s_{2}^{a}}^{s_{2}^{b}},
$$

for $\left|\boldsymbol{k}_{a, b}\right|<k_{\mathrm{th}}$. It is easy to see that this $U(\boldsymbol{x}, \boldsymbol{y})$ is energy $(\boldsymbol{k})$ independent by construction and satisfies eq. $(9)$ as

$$
\begin{gathered}
\sum_{\Gamma_{b}} \int d^{3} y U_{\Gamma, \Gamma_{a}}(\boldsymbol{x}, \boldsymbol{y}) \Psi_{\Gamma_{a}}^{A}(\boldsymbol{y})=\sum_{A_{a}, A_{b}}^{\left|\boldsymbol{k}_{a, b}\right|<k_{\mathrm{th}}}\left[E_{k_{a}}-H_{0}\right] \\
\times \Psi_{\Gamma}^{A_{a}}(\boldsymbol{x}) \mathcal{N}_{A_{a}, A_{b}}^{-1} \mathcal{N}^{A_{b}, A}=\left[E_{k}-H_{0}\right] \Psi_{\Gamma}^{A}(\boldsymbol{x})
\end{gathered}
$$

for $|\boldsymbol{k}|<k_{\mathrm{th}}$. Once the non-local potential $U(\boldsymbol{x}, \boldsymbol{y})$ which satisfies eq. (9) is obtained, we can extract the phase shift $\delta_{L S I}(k)$ at $k<k_{\mathrm{th}}$ in QCD under the property in eq. (8), by solving the Schrödinger equation with this potential. Since the potential itself is not a physical observable, however, the potential which satisfies eq. $(9)$ is not unique. One may add terms which affect eq. (9) only above the inelastic threshold $\left(k>k_{\mathrm{th}}\right)$ while keeping eq. (9) intact below the inelastic threshold.

\subsection{Velocity expansion}

The construction of $U(\boldsymbol{x}, \boldsymbol{y})$ given in eq. 10$)$ is important to prove that such a energy-independent potential indeed exists. In lattice QCD simulations, however, the NBS wave 
functions can be obtained only for a ground state and possibly a few low-lying excited states, so that a summation over $A_{a}$ becomes inaccurate. For practical uses, we therefore expand the non-local potential in terms of velocity (or derivative) and determine local coefficient functions order by order of the expansion [4,5, 6]. Explicitly we have $U(\boldsymbol{x}, \boldsymbol{y})=V(\boldsymbol{x}, \nabla) \delta^{(3)}(\boldsymbol{x}-\boldsymbol{y})$ with

$$
\begin{aligned}
V(\boldsymbol{x}, \nabla) & =\underbrace{V_{0}(r)+V_{\sigma}(r) \boldsymbol{\sigma}_{1} \cdot \boldsymbol{\sigma}_{2}+V_{T}(r) S_{12}}_{=V^{\mathrm{LO}}(\boldsymbol{x})} \\
& +\underbrace{V_{\mathrm{LS}}(r) \boldsymbol{L} \cdot \boldsymbol{S}}_{=V^{\mathrm{NLO}}(\boldsymbol{x})}+O\left(\nabla^{2}\right), \quad r=|\boldsymbol{x}|,
\end{aligned}
$$

where $\boldsymbol{\sigma}_{i}$ is the Pauli-matrix acting on the spin index of the $i$-th nucleon, $S_{12}=3\left(\boldsymbol{x} \cdot \boldsymbol{\sigma}_{1}\right)\left(\boldsymbol{x} \cdot \boldsymbol{\sigma}_{2}\right) / r^{2}-\boldsymbol{\sigma}_{1} \cdot \boldsymbol{\sigma}_{2}$ is the tensor operator, $\boldsymbol{L}=\boldsymbol{x} \times \boldsymbol{p}$ is an orbital angular momentum, and $\boldsymbol{S}=\left(\boldsymbol{\sigma}_{1}+\boldsymbol{\sigma}_{2}\right) / 2$ is the total spin. Each local coefficient function is further decomposed as $V_{X}(r)=$ $V_{X}^{0}(r)+V_{X}^{\tau}(r) \boldsymbol{\tau}_{1} \cdot \boldsymbol{\tau}_{2}$ for $X=0, \sigma, T, \operatorname{LS}, \cdots$, where $\boldsymbol{\tau}_{i}$ is the flavor matrix acting on the flavor index of the $i$-th nucleon. This form of the velocity expansion agrees with the result obtained by the symmetry 23$]$.

We can determine these coefficient functions from NBS wave functions with particular quantum numbers, order by order in the velocity expansion. Once the potential is obtained at some order of the expansion, we can solve the Schrödinger equation with this potential in order to extract physical observables such as the scattering phase shift and the binding energy of the deuteron.

\subsection{Remarks}

Let us give some remarks on the HAL QCD method.

First of all, potentials themselves are not physical observables, and they are therefore not unique. For example, potentials depend on the choice of nucleon operators to define the NBS wave functions. We adopt here the local nucleon operator where all three quarks are put on the same point, since it is a convenient choice for the reduction formula of composite particles $24,25,26$, but other choices are equally possible. We consider such a dependence of a potential on its definition as a "scheme" of the potential. While the potential is therefore scheme dependent, physical observables such as the scattering phase shift and the binding energy are of course physical thus scheme independent.

Although potentials are scheme dependent, they are useful to understand or describe "physics" of hadronic interactions. For example, the $N N$ potential best summarizes the $N N$ scattering at higher energy in terms of its repulsive core, as the running coupling constant in QCD, which is also scheme dependent, describes the deep inelastic scattering data at high energy well in terms of its asymptotic freedom.

Among different schemes (of potentials or running couplings constant), some schemes are better than others. While a good convergence of the perturbative expansion for a certain class of observables is a reasonable criteria for a good running coupling constant, a good convergence of the velocity expansion, which means weak non-locality, is a reasonable criteria for a good potential. In this sense, a completely local and energy-independent potential would be the best one if no inelastic threshold were present.

It is also important to note that the convergence of the velocity expansion of the potential can be examined within the HAL QCD method. For example, if we have $\Psi^{A_{n}}$ for $n=1,2, \cdots, N$, from which we can determine $N-1$ local functions in the velocity expansion in $N$ different ways. A variation among $N$ different ways gives an estimate of the size of higher order terms neglected. Furthermore we can determine one of these higher order terms using all $N$ NBS wave functions. A convergence of the velocity expansion will be considered later.

\section{Lattice methods}

In this section, we consider an explicit procedure to extract potentials in lattice QCD simulations.

\subsection{An extraction of NBS wave functions from correlation functions}

We first consider a method to extract NBS wave functions from 4-pt correlation functions, defined by

$$
F_{\Gamma}\left(\boldsymbol{x}, t-t_{0}\right)=\left\langle 0\left|T\left\{N_{\alpha, f}(\boldsymbol{r}, t) N_{\beta, g}(\boldsymbol{r}+\boldsymbol{x}, t)\right\} \overline{\mathcal{J}}\left(t_{0}\right)\right| 0\right\rangle
$$

for $t>t_{0}$, where $\Gamma=\alpha \beta, f g$, and $\overline{\mathcal{J}}\left(t_{0}\right)$ is a source operator which creates two-nucleon states and its explicit form will be given later. By inserting a complete set in the above definition, we obtain

$$
\begin{aligned}
& F_{\Gamma}\left(\boldsymbol{x}, t-t_{0}\right)=\langle 0| T\left\{N_{\alpha, f}(\boldsymbol{r}, t) N_{\beta, g}(\boldsymbol{r}+\boldsymbol{x}, t)\right\} \\
& \sum_{n, s_{1} s_{2}}\left|N N, A_{n}\right\rangle_{\text {in }} \times_{\text {in }}\left\langle N N, A_{n}\left|\overline{\mathcal{J}}\left(t_{0}\right)\right| 0\right\rangle+\cdots \\
= & \sum_{n, s_{1} s_{2}} Z\left(A_{n}\right) \Psi_{\Gamma}^{A_{n}}(\boldsymbol{x}) e^{-W_{\boldsymbol{k}_{n}}\left(t-t_{0}\right)}+\cdots,
\end{aligned}
$$

where $A_{n}=\boldsymbol{k}_{n}, s_{1} s_{2}, Z\left(A_{n}\right)={ }_{\text {in }}\left\langle N N, A_{n}|\overline{\mathcal{J}}(0)| 0\right\rangle$, and ellipses represent inelastic contributions.

As in the standard method to extract hadron masses in lattice QCD, we extract the NBS wave function for the ground state from the above correlation function by taking $\left(t-t_{0}\right) \rightarrow \infty$ as

$$
F_{\Gamma}\left(\boldsymbol{x}, t-t_{0}\right) \simeq Z\left(A_{0}\right) \Psi_{\Gamma}^{A_{0}}(\boldsymbol{x}) e^{-W_{\boldsymbol{k}_{0}}\left(t-t_{0}\right)},
$$

where $W_{\boldsymbol{k}_{0}}$ is the lowest energy of $N N$ states.

The extraction of the NBS wave function in eq. (14) relies on the ground state saturation for the correlation function, which can in principle be achieved by taking a large $t-t_{0}$. In practice, however, it is difficult to realize the ground state saturation for the $N N$ system within reasonable errors, since $F_{\Gamma}\left(\boldsymbol{x}, t-t_{0}\right)$ becomes very noisy at large $t-t_{0}$. 


\subsection{An improved extraction}

The signal-to-noise ratio for the nucleon 4-pt function $F_{\Gamma}$ behaves for large $t$ as $\mathcal{S} / \mathcal{N} \sim e^{-2\left(m_{N}-3 m_{\pi} / 2\right) t}$ [27, 28], which decreases for lighter pion masses toward its physical value. Furthermore, as we increase the volume, the splitting between the ground state and the 1st excited state for the $N N$ system becomes smaller as $\Delta E \simeq \boldsymbol{k}_{\min }^{2} / m_{N}=$ $(2 \pi / L)^{2} / m_{N}$, which requires lager $t$ for the ground state saturation. The behavior of statistical noise in the above, however, makes the signals very poor at such large $t$ for the $N N$ system.

An improved extraction of the NBS wave function has recently been proposed to overcome the above difficulties 29 . We first normalize the 4-point correlation function as

$$
R_{\Gamma}(\boldsymbol{x}, t) \equiv \frac{F_{\Gamma}(\boldsymbol{x}, t)}{\left(e^{-m_{N} t}\right)^{2}} \simeq \sum_{n, s_{1}, s_{2}} Z\left(A_{n}\right) \Psi_{\Gamma}^{A_{n}}(\boldsymbol{x}) e^{-\Delta W_{\boldsymbol{k}_{n}} t}
$$

where $\Delta W_{\boldsymbol{k}}=2 \sqrt{m_{N}^{2}+\boldsymbol{k}^{2}}-2 m_{N}$. Using an identity $\Delta W_{\boldsymbol{k}}=\boldsymbol{k}^{2} / m_{N}-\left(\Delta W_{\boldsymbol{k}}\right)^{2} /\left(4 m_{N}\right)$ and neglecting inelastic contributions, we obtain the time-dependent Schrödingerlike equation

$$
\begin{aligned}
& \left\{-H_{0}-\frac{\partial}{\partial t}+\frac{1}{4 m_{N}} \frac{\partial^{2}}{\partial t^{2}}\right\} R_{\Gamma}(\boldsymbol{x}, t) \\
= & \sum_{\Gamma_{a}} \int d^{3} y U_{\Gamma \Gamma_{a}}(\boldsymbol{x}, \boldsymbol{y}) R_{\Gamma_{a}}(\boldsymbol{y}, t) \\
\simeq & \sum_{\Gamma_{a}} V_{\Gamma \Gamma_{a}}(\boldsymbol{x}) R_{\Gamma_{a}}(\boldsymbol{x}, t)+\cdots,
\end{aligned}
$$

which shows that the same $U(\boldsymbol{x}, \boldsymbol{y})$ in eq. 9 can be obtained from $R_{\Gamma}(\boldsymbol{x}, t)$. An advantage of this method is that the ground state saturation is no more required for $R_{\Gamma}(\boldsymbol{x}, t)$ to satisfy eq. (15) or eq. (16). For this method to work, however, $t$ has to be large enough that elastic contributions dominate $R_{\Gamma}(\boldsymbol{x}, t)$.

In the scattering of two different particles such as the $N \Xi$ scattering, we must employ the non-relativistic expansion as $\Delta W_{\boldsymbol{k}}=\sqrt{\boldsymbol{k}^{2}+m_{N}^{2}}+\sqrt{\boldsymbol{k}^{2}+m_{\Xi}^{2}}-m_{N}-m_{\Xi} \simeq$ $\boldsymbol{k}^{2} /(2 \mu)$ with the reduced mass $\mu=m_{N} m_{\Xi} /\left(m_{N}+m_{\Xi}\right)$. Within this approximation, we obtain

$$
\left\{-H_{0}-\frac{\partial}{\partial t}\right\} R_{\Gamma}^{N \Xi}(\boldsymbol{x}, t) \simeq \sum_{\Gamma_{a}} V_{\Gamma \Gamma_{a}}(\boldsymbol{x}) R_{\Gamma_{a}}^{N \Xi}(\boldsymbol{y}, t)
$$

\subsection{Source operators}

We choose the source operator $\mathcal{J}$ so as to fix quantum numbers of the state $\left|N N, A_{n}\right\rangle$. Instead of the $S O(3, \boldsymbol{R})$, we classify the states on the hyper cubic lattice in terms of the irreducible representation of the cubic transformation $S O(3, \boldsymbol{Z})$, denoted by $A_{1}, A_{2}, E, T_{1}, T_{2}$, whose dimensions are $1,1,2,3,3$. In Table 1 the orbital angular momentum $L$ representation in $S O(3, \boldsymbol{R})$ is decomposed in terms
Table 1. A decomposition of irreducible representations of $S O(3, \boldsymbol{R})$ with the orbital angular momentum $L$ in terms of $S O(3, \boldsymbol{Z})$ representations. Here $P=(-1)^{L}$ represents parity.

\begin{tabular}{lllllll}
\hline$L$ & $P$ & $A_{1}$ & $A_{2}$ & $E$ & $T_{1}$ & $T_{2}$ \\
\hline $0(S)$ & + & 1 & 0 & 0 & 0 & 0 \\
$1(P)$ & - & 0 & 0 & 0 & 1 & 0 \\
$2(D)$ & + & 0 & 0 & 1 & 0 & 1 \\
$3(F)$ & - & 0 & 1 & 0 & 1 & 1 \\
$4(G)$ & + & 1 & 0 & 1 & 1 & 1 \\
$5(H)$ & - & 0 & 0 & 1 & 2 & 1 \\
$6(I)$ & + & 1 & 1 & 1 & 1 & 2 \\
\hline
\end{tabular}

Table 2. A decomposition of the direct product $R_{1} \otimes R_{2}$ in terms of the direct sum of irreducible representations. By definition $R_{1} \otimes R_{2}=R_{2} \otimes R_{1}$.

\begin{tabular}{cc|c||cl|c}
\hline$R_{1}$ & $R_{2}$ & $R_{1} \otimes R_{2}$ & $R_{1}$ & $R_{2}$ & $R_{1} \otimes R_{2}$ \\
\hline$A_{1}$ & $A_{1}$ & $A_{1}$ & $E$ & $E$ & $A_{1} \oplus A_{2} \oplus E$ \\
$A_{1}$ & $A_{2}$ & $A_{2}$ & $E$ & $T_{1}$ & $T_{1} \oplus T_{2}$ \\
$A_{1}$ & $E$ & $E$ & $E$ & $T_{2}$ & $T_{1} \oplus T_{2}$ \\
$A_{1}$ & $T_{1}$ & $T_{1}$ & $T_{1}$ & $T_{1}$ & $A_{1} \oplus E \oplus T_{1} \oplus T_{2}$ \\
$A_{1}$ & $T_{2}$ & $T_{2}$ & $T_{1}$ & $T_{2}$ & $A_{2} \oplus E \oplus T_{1} \oplus T_{2}$ \\
$A_{2}$ & $A_{2}$ & $A_{1}$ & $T_{2}$ & $T_{2}$ & $A_{1} \oplus E \oplus T_{1} \oplus T_{2}$ \\
$A_{2}$ & $E$ & $E$ & & & \\
$A_{2}$ & $T_{1}$ & $T_{2}$ & & & \\
$A_{2}$ & $T_{2}$ & $T_{1}$ & & & \\
\hline
\end{tabular}

of irreducible representations in $S O(3, \boldsymbol{Z})$ at $L \leq 6$. For example, the table says that the source operator $\mathcal{J}\left(t_{0}\right)$ in the $A_{1}$ representation with positive parity produces states with $L=0,4,6, \cdots$ at $t=t_{0}$ from the vacuum, while that in the $T_{1}$ with negative parity produces states with $L=1,3,5, \cdots$. The total spin $S$ for two nucleons becomes $1 / 2 \otimes 1 / 2=1 \oplus 0$, corresponding to $T_{1}(S=1)$ and $A_{1}(S=0)$ of the $S O(3, \boldsymbol{Z})$, respectively. Thus the total "angular momentum" $J$ for a two nucleon system is given by the product of $R_{1} \otimes R_{2}$, where $R_{1}=A_{1}, A_{2}, E, T_{1}, T_{2}$ for the orbital "angular momentum" while $R_{2}=A_{1}, T_{1}$ for the total spin. In Table 2 , a decomposition of the product $R_{1} \otimes R_{2}$ is given in terms of the direct sum.

Most of the HAL QCD results are obtained by the wall source defined by

$$
\mathcal{J}_{\alpha \beta, f g}^{\text {wall }}(t)=N_{\alpha, f}^{\text {wall }}(t) N_{\beta, g}^{\text {wall }}(t),
$$

where $\alpha, \beta=1,2$ are (upper) spinor indices, $f, g$ are flavor indices, and $N^{\text {wall }}(t)$ is obtained by replacing local quark field $q(x)$ of $N(x)$ with the wall quark field, $q^{\text {wall }}(t) \equiv$ $\sum_{\boldsymbol{x}} q(\boldsymbol{x}, t)$ together with the Coulomb gauge fixing only at the time slice of the source.

The source operator $\overline{\mathcal{J}}^{\text {wall }}\left(t_{0}\right)$ creates states with the zero angular momentum at $t=t_{0}$, which belongs to the $A_{1}$ representation with positive parity $P=+$. Using the spin projection $P^{(S)}$ with $P^{S=0}=\sigma_{2}, P^{S=1}=\sigma_{1}$, we fix the total angular momentum $J$ with $J_{z}=0$ and the total 
isospin $I$ of the source as

$$
\mathcal{J}\left(t_{0} ; J^{P=+}, I\right)=P_{\beta \alpha}^{(S)} \mathcal{J}_{\alpha \beta, f g}\left(t_{0}\right),
$$

where the total isospin $I$ is automatically fixed once the total spin is given due to the fermonic nature of nucleons: $(S, I)=(0,1)$ or $(1,0)$. Since $A_{1}^{+}(L=0) \otimes A_{1}(S=$ $0)=A_{1}^{+}$or $A_{1}^{+}(L=0) \otimes T_{1}(S=1)=T_{1}^{+}$, the state with $\left(J^{P}, I\right)=\left(A_{1}^{+}, 1\right)$ for the spin-singlet or $\left(J^{P}, I\right)=\left(T_{1}^{+}, 0\right)$ for the spin-triplet is created at $t=t_{0}$ by the above source. As these quantum numbers are conserved by QCD interactions, the NBS wave function extracted at $t>t_{0}$ has the same $\left(J^{P}, I\right)$. Moreover, as a speciality of the two nucleon system with equal up and down quark masses, the total spin $S$ is also conserved at $t>t_{0}$ : The constraint $(-1)^{(S+1)+(I+1)} P=-1$ should be satisfied due to the fermionic nature of nucleons, while parity $P$ and the isospin $I$ are conserved in QCD with equal up and down quark masses. Therefore $S$ is conserved under the condition that $S=0,1$. The orbital angular momentum $L$, however, is not conserved in general. While the state with $\left(J^{P}, I\right)=\left(A_{1}^{+}, 1\right)$ has $L=A_{1}^{+}$even at $t>t_{0}$, the other state with $\left(J^{P}, I\right)=\left(T_{1}^{+}, 0\right)$ has $L=A_{1}^{+}$and $L=E^{+} \oplus T_{2}^{+}$components at $t>t_{0}$, corresponding to $L=0$ (S-wave) and $L=2$ (D-wave) in $S O(3, \boldsymbol{R})$. Note that Table 2 tells us that not only $L=A_{1}^{+}, E^{+} \oplus T_{2}^{+}$components but also the $L=T_{1}^{+}$component exist in the state with $\left(J^{P}, I\right)=\left(T_{1}^{+}, 0\right)$. The latter extra component is expected to be small since it appears as a consequence of the violation of rotational symmetry on the cubic lattice.

The orbital angular momentum $L$ of the NBS wave function can be projected onto a particular value by the operator $P^{(L)}$ as

$$
\Psi^{A}\left(\boldsymbol{x} ; J^{P}, I, L\right)=P^{(L)} \Psi^{A}\left(\boldsymbol{x} ; J^{P}, I\right)
$$

where the total spin is given by $S=1-I$, and $\Psi^{A}\left(\boldsymbol{x} ; J^{P}, I\right)$ is extracted from the 4-pt function generated by the source $\mathcal{J}\left(t_{0} ; J^{P}, I\right)$ as

$$
\begin{aligned}
F_{\Gamma}\left(\boldsymbol{x}, t-t_{0} ; J^{P}, I\right) & \simeq Z\left(A, J^{P}, I\right) \Psi_{\Gamma}^{A}\left(\boldsymbol{x} ; J^{P}, I\right) e^{-W_{\boldsymbol{k}}\left(t-t_{0}\right)}, \\
Z\left(A, J^{P}, I\right) & ={ }_{\mathrm{in}}\left\langle N N, A\left|\overline{\mathcal{J}}\left(0 ; J^{P}, I\right)\right| 0\right\rangle
\end{aligned}
$$

for large $t-t_{0}$. The projection $P^{(L)}$ is defined for an arbitrary function $\varphi(\boldsymbol{x})$ by

$$
P^{(L)} \varphi(\boldsymbol{x})=\frac{d_{L}}{24} \sum_{g \in S O(3, \boldsymbol{Z})} \chi^{L}(g) \varphi\left(g^{-1} \boldsymbol{x}\right)
$$

for $L=A_{1}, A_{2}, E, T_{1}, T_{2}$, where $\chi^{L}$ denotes the character for $L, g$ is one of 24 elements of $S O(3, \boldsymbol{Z})$ and $d_{L}$ is the dimensions of $L$. It is also noted that the $A_{1}$ state may contain $L=4,6, \cdots$ components other than the dominant $L=0$ component.

\subsection{Local potentials at the leading order}

Local potentials at the leading order ( $\mathrm{LO}$ ) of the velocity expansion take the form

$$
V^{\mathrm{LO}}(r)=V_{0}(r)+V_{\sigma}(r) \boldsymbol{\sigma}_{1} \cdot \boldsymbol{\sigma}_{2}+V_{T}(r) S_{12} .
$$

For the isospin-triplet (spin-singlet) sector, $S_{12}=0$ and $\boldsymbol{\sigma}_{1} \cdot \boldsymbol{\sigma}_{2}=-3$ imply

$$
V_{C}^{I=1}(r)=\frac{\left[E_{k}-H_{0}\right] \Psi^{A}\left(\boldsymbol{x} ; A_{1}^{+}, 1\right)}{\Psi^{A}\left(\boldsymbol{x} ; A_{1}^{+}, 1\right)},
$$

where $V_{C}^{I=1}(r)=V_{0}^{I=1}(r)-3 V_{\sigma}^{I=1}(r)$, which is often referred to as the central potential for the ${ }^{1} S_{0}$ state, where the notation ${ }^{2 S+1} L_{J}$ is used. It is noted, however, that the potentials at the LO of the velocity expansion do not depend on the quantum numbers of the state, $J$ and $L$. In this sense, it is more precise to say that $V_{C}^{I=1}(r)$ is the parity-even isospin-triplet (spin-singlet) potential determined from the state with $J=L=A_{1}$. Determinations of this potential from other states can give estimates for the size of contributions from higher order terms in the velocity expansion.

For the isospin-singlet (spin-triplet) sector, both tensor potential $V_{T}$ and central potential $V_{C}$ appear at the LO. The Schrödinger equation for the state with $\left(J^{P}, I\right)=$ $\left(T_{1}^{+}, 0\right)$ reads

$$
\begin{aligned}
& {\left[H_{0}+V_{C}^{I=0}(r)+V_{T}^{I=0}(r) S_{12}\right] \Psi^{A}\left(\boldsymbol{x} ; T_{1}^{+}, 0\right) } \\
= & E_{k} \Psi^{A}\left(\boldsymbol{x} ; T_{1}^{+}, 0\right),
\end{aligned}
$$

where $V_{C}^{I=0}(r)=V_{0}^{I=0}(r)+V_{\sigma}^{I=0}(r)$. With projections onto $A_{1}$ and $E \oplus T_{2}$ components, we have $V_{C}^{I=0}$ and $V_{T}^{I=0}$ as

$$
\begin{aligned}
V_{C}^{I=0}(r) & =E_{k}-\frac{1}{\Delta(\boldsymbol{x})}\left(\left[\mathcal{Q} S_{12} \Psi^{A}\right](\boldsymbol{x}) H_{0}\left[\mathcal{P} \Psi^{A}\right](\boldsymbol{x})\right. \\
& \left.-\left[\mathcal{P} S_{12} \Psi^{A}\right](\boldsymbol{x}) H_{0}\left[\mathcal{Q} \Psi^{A}\right](\boldsymbol{x})\right), \\
V_{T}^{I=0}(r) & =\frac{1}{\Delta(\boldsymbol{x})}\left(\left[\mathcal{Q} \Psi^{A}\right](\boldsymbol{x}) H_{0}\left[\mathcal{P} \Psi^{A}\right](\boldsymbol{x})\right. \\
& \left.-\left[\mathcal{P} \Psi^{A}\right](\boldsymbol{x}) H_{0}\left[\mathcal{Q} \Psi^{A}\right](\boldsymbol{x})\right),
\end{aligned}
$$

$\Delta(\boldsymbol{x}) \equiv\left[\mathcal{Q}_{12} \Psi^{A}\right](\boldsymbol{x})\left[\mathcal{P} \Psi^{A}\right](\boldsymbol{x})-\left[\mathcal{P} S_{12} \Psi^{A}\right](\boldsymbol{x})\left[\mathcal{Q} \Psi^{A}\right](\boldsymbol{x})$,

where $\mathcal{P} \Psi^{A}(\boldsymbol{x})=P^{\left(A_{1}\right)} f(\boldsymbol{x})$ and $\mathcal{Q} \Psi^{A}(\boldsymbol{x})=P^{\left(E \oplus T_{2}\right)} f(\boldsymbol{x})$ with $f(\boldsymbol{x}) \equiv \Psi_{\Gamma}^{A}\left(\boldsymbol{x} ; T_{1}^{+}, 0\right)$. In numerical simulations, $\Gamma=$ $(\alpha \beta, f g)=(2,1,2,1)$ is mainly employed, and the approximation that $\mathcal{Q} \simeq 1-\mathcal{P}$ is used by neglecting small $T_{1}$ component. We may define the effective central potential as

$$
V_{C, \mathrm{eff}}^{I=0}(r)=\frac{\left[E_{k}-H_{0}\right] \mathcal{P} \Psi^{A}(\boldsymbol{x})}{\mathcal{P} \Psi^{A}(\boldsymbol{x})},
$$

which differs from $V_{C}^{I=0}(r)$ by $O\left(V_{T}^{2}\right)$ in the second order perturbation for small $V_{T}$.

\subsection{A comparison}

We here briefly compare the potential method with the direct extraction of the phase shift via the Lüsher's finite volume method in lattice QCD. 
The potential method by construction gives the correct phase shift at a particular $k$ where the NBS wave function is calculated, while the phase shift at other values of $k$ is approximated one due to the velocity expansion of the non-local potential. With this systematic uncertainty in mind, the potential method can reveal the global structure of the phase shift in the wide range of (continuous) $k$, while the finite volume method can gives the exact phase shift at a few discrete points of $k$.

The finite size correction to the potential is expected to be small, since no massless particle exchange exists between nucleons. Indeed, the Lüsher's finite volume method assumes that the potential remains intact as long as the volume is large enough so that the interaction range of the potential is smaller then the half of the lattice extension, $L / 2$. Under this condition, the scattering wave satisfies the free Schrödinger equation outside the interaction range. Due to the (periodic) boundary condition, we have the discrete values of $k$ in the finite box, which give some information of hadronic interactions 2. To extract the phase shift at the corresponding values of $k$, we need an additional assumption: Let us consider the allowed values of $k$ for the state with $L=A_{1}$, which contains not only $L=0$ components but also $L=4,6, \cdots$, contributions. We then extract the phase shift $\delta_{L}(k)$ for the $L=0$ partial wave, under the assumption that the $L=0$ component dominates in this state. In the case of the potential method, on the other hand, we do not need such an assumption. Once the potential is obtained, we can calculate the phase shift for an arbitrary $L$ by solving the Schrödinger equation in the infinite volume, again with the systematic uncertainty of the velocity expansion.

We also expect that the quark mass dependence of the potential is much milder than that of physical observables such as the scattering length. While the $N N$ scattering length is small in the heavy quark mass region, it diverges when the deuteron bound state is formed at a lighter quark mass 30 . Therefore the scattering length varies from almost zero to infinity as the quark mass decreases. Such a drastic change of the scattering length can be realized by a small change of the potential shape as a function of the quark mass. This would make chiral extrapolations for the potentials to the physical pion mass more stable than those for the scattering length.

\section{Lattice results}

In this section, numerical results for nuclear potentials obtained by the HAL QCD collaboration are introduced.

\subsection{Nuclear potentials in full QCD by the improved extraction}

We evaluate nuclear potentials, employing (2+1)-flavor QCD gauge configurations generated by PACS-CS collaboration 31 on $32^{3} \times 64$ lattice with the RG improved Iwasaki gauge action at $\beta=1.9$ and the non-perturbatively $O(a)$ improved Wilson quark action with $C_{\mathrm{SW}}=1.715$,

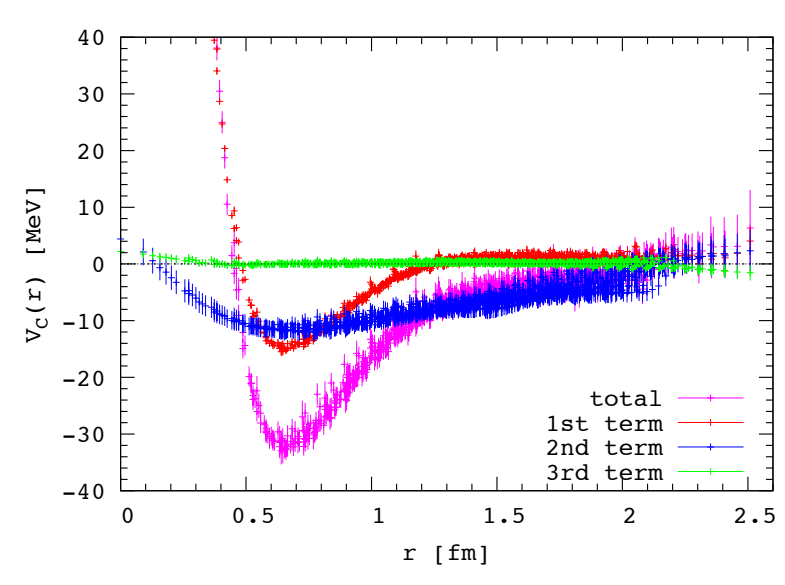

Fig. 1. Three contributions to $V_{C}^{I=1}(r)$ in eq. 29 at $t=9$. Taken from Ref. 29].

which corresponds to the lattice spacing $a \simeq 0.091 \mathrm{fm}$ $\left(a^{-1}=2.176(31) \mathrm{GeV}\right)$, the spatial extent $L=32 a \simeq 2.90$ fm. We calculate $R(\boldsymbol{x}, t)$ at a fixed value of light and strange quark mass combination, which corresponds to $m_{\pi} \simeq 701 \mathrm{MeV}, m_{K} \simeq 789 \mathrm{MeV}$ and $m_{N} \simeq 1583 \mathrm{MeV}$.

The periodic boundary condition is used for spatial directions, while the Dirichlet boundary condition is taken for the temporal direction at $t_{\mathrm{DBC}}=32 a$ and $-32 a$, to avoid opposite propagations of two nucleons in temporal direction, i.e, one propagates forward and the other propagates backward. From time-reversal and charge conjugation symmetries, we can average over forward propagation at $t>0$ and backward propagation at $t<0$ with a wall source at $t=0$. By temporally shifting gauge configurations, 21 source points are used per one configuration and 390 gauge configurations are employed in total. Statistical errors are estimated by the Jackknife method with a bin size of 10 configurations. In our actual calculation, we replace $e^{-m_{N} t}$ in the denominator of $R(\boldsymbol{x}, t)$ by the singlenucleon propagator $C_{N}(t) \equiv \sum_{\boldsymbol{x}}\langle 0|T[N(x) \bar{N}(0)]| 0\rangle$. Time derivatives are evaluated after applying the polynomial interpolation of degree 5 to $R(\boldsymbol{x}, t)$.

In the improved extraction, the central potential $V_{C}^{I=1}(r)$ at the $\mathrm{LO}$ is given by

$$
\begin{aligned}
V_{C}^{I=0}(r) & =-\frac{H_{0} R(\boldsymbol{x}, t)}{R(\boldsymbol{x}, t ;)}-\frac{(\partial / \partial t) R(\boldsymbol{x}, t)}{R(\boldsymbol{x}, t)} \\
& +\frac{1}{4 m_{N}} \frac{(\partial / \partial t)^{2} R(\boldsymbol{x}, t)}{R(\boldsymbol{x}, t)}
\end{aligned}
$$

which are shown separately in Fig. 1 at $t=929$. The first term in eq. 29) (the red points) determines the overall shape of the potential, while the second term (the blue points) gives a major correction. The third term (the green points) corresponding to the relativistic correction, on the other hand, is negligible, showing that the non-relativistic approximation $\Delta W_{\boldsymbol{k}} \simeq \boldsymbol{k}^{2} / m_{N}$ works well. Note that the second term in eq. 29 would be constant if the ground 


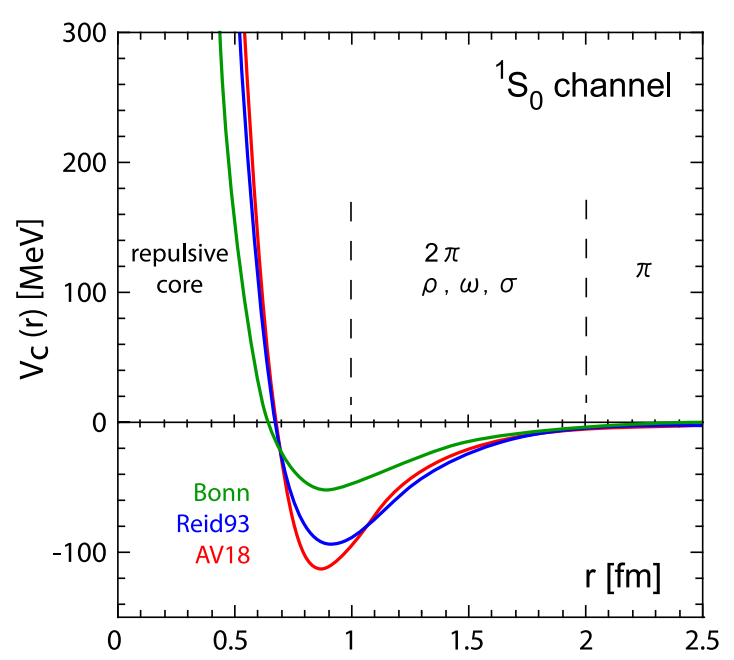

Fig. 2. Three examples of the phenomenological $N N$ potential in the isospin-triplet (spin-singlet) sector $\left(V_{C}^{I=1}(r)\right)$, Bonn 32 , Reid93 33] and Argonne $v_{18}$ 34. Taken from Ref. 4].

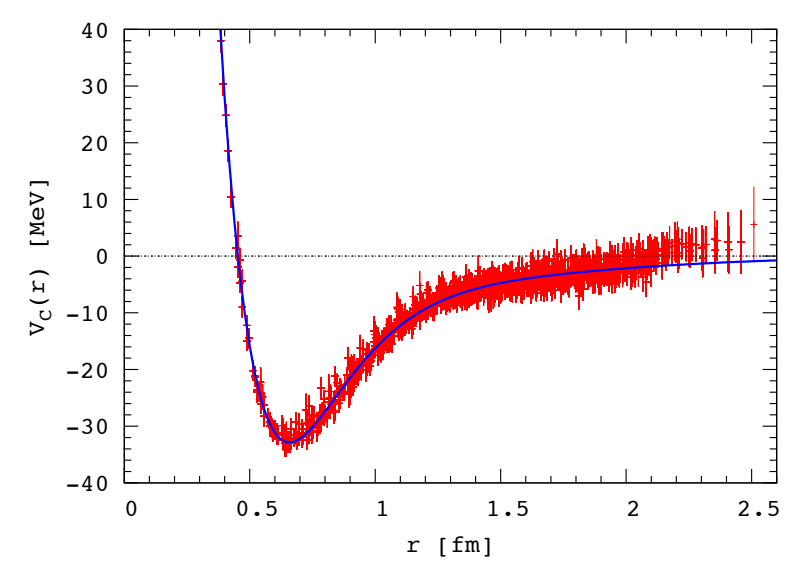

Fig. 3. The multi-Gaussian fit of the central potential $V_{C}^{I=1}(r)$ with $N_{\text {Gauss }}=5$ at $t=9$. Taken from Ref. [29].

state saturation were achieved. A clear $r$-dependence of the second term tells us that contaminations of excited states indeed exist and are non-negligible.

The potential in Fig. 1 has a similar structure to the know phenomenological $N N$ potentials, namely the repulsive core at short distance surrounded by the attractive well at medium and long distances, as shown in Fig. 2 The first result for the NN potential, obtained in quenched QCD by the HAL QCD method [4, also reproduces this structure, and this success has received general recognition 35 Note however that lattice artifacts may be large at very short distance (i.e. $r \leq 0.1 \mathrm{fm}$ ).

\subsection{Scattering phase shift}

To calculate the $N N$ scattering phase shift by solving the Schrödinger equation with the potential in the infinite vol-

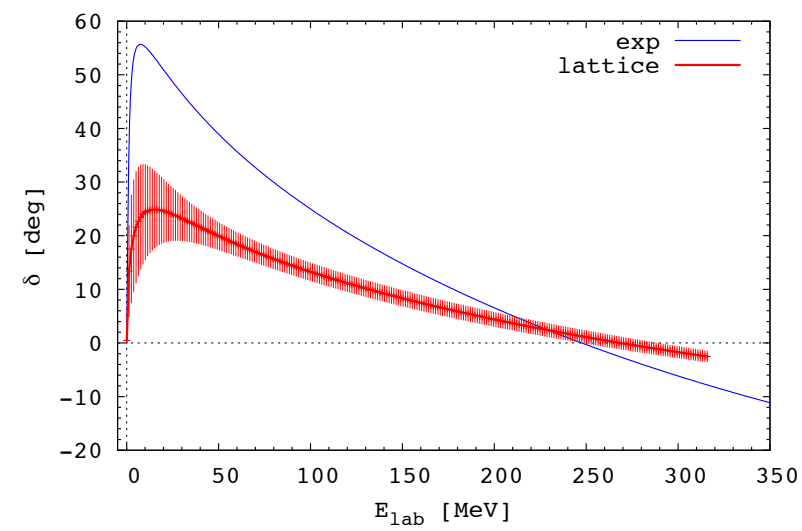

Fig. 4. The scattering phase in ${ }^{1} S_{0}$ channel in the laboratory frame obtained from the lattice $N N$ potential, together with experimental data 36]. Taken from Ref. [29].

ume, the central potential $V_{C}^{I=1}(r)$ is fitted with multiGaussian functions as $g(r) \equiv \sum_{n=1}^{N_{\text {gauss }}} V_{n} \cdot \exp \left(-\nu_{n} r^{2}\right)$, where $V_{n}$ and $\nu_{n}(>0)$ are used as fit parameters, $N_{\text {gauss }}$ denotes the number of Gaussian functions. The fit with multi-Gaussians but without a Yukawa-function works well, as shown in Fig. 329, presumably due to the heavy pion mass.

We then solve the Schrödinger equation in ${ }^{1} S_{0}$ channel with this potential, in order to extract the scattering phase $\delta(k)$, which is shown in Fig. 4 together with the experimental data for comparison[29]. Qualitative feature of the phase shift as a function of $k$ is well reproduced, though the strength is weaker, most likely due to the heavy pion mass $\left(m_{\pi} \simeq 701 \mathrm{MeV}\right)$ in this calculation. In fact, the recent 3-flavor QCD simulations show that the $N N$ phase shift approaches toward the physical value as the quark mass decreases 11. The scattering length at $m_{\pi} \simeq 701$ $\mathrm{MeV}$ in the present method, calculated from the derivative of the scattering phase shift at $E_{\text {lab }}=0$, leads to $a\left({ }^{1} S_{0}\right)=\lim _{k \rightarrow 0} \tan \delta(k) / k=1.6 \pm 1.1 \mathrm{fm}$, which is still smaller than the experimental value at the physical point, $a^{(\exp )}\left({ }^{1} S_{0}\right) \sim 20 \mathrm{fm}$ ( strong attractive in our sign convention), as seen from a comparison in Fig. 4 .

\subsection{Tensor potential}

35 Jsing the same gauge configurations generated by the PACS-CS collaboration, the LO potentials for the isospinsinglet (spin-triplet) have been extracted. In Fig. 5 we show $V_{C}^{I=0}(r)$ and $V_{T}^{I=0}(r)$, together with $V_{C}^{I=1}(r)$ for a comparison [37. While central potentials for both sectors look similar, the tensor potential $V_{T}^{I=0}(r)$ is negative for the whole range of $r$, so that no repulsive core appears in this sector. The tensor potential seems finite at $r=0$, but we have to be careful to conclude such a short distance 


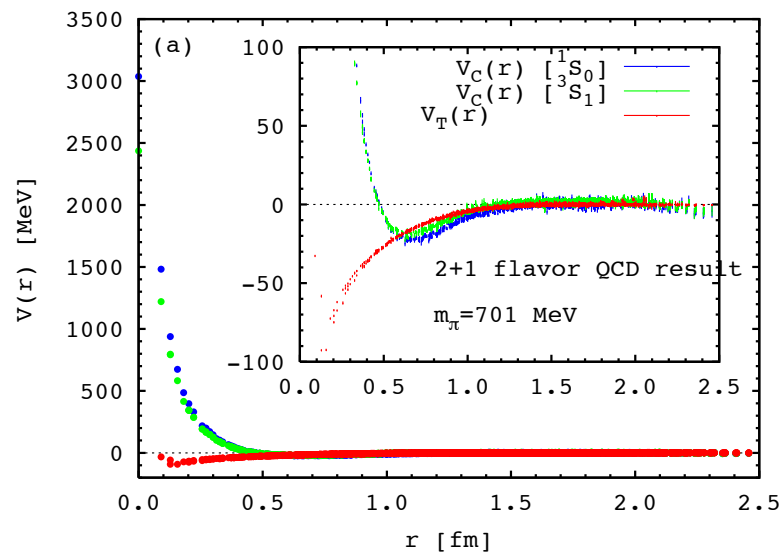

Fig. 5. Central and tensor potentials in $2+1$ flavor QCD at $m_{\pi} \simeq 701 \mathrm{MeV}$ by the improved method. Taken from Ref. 37.

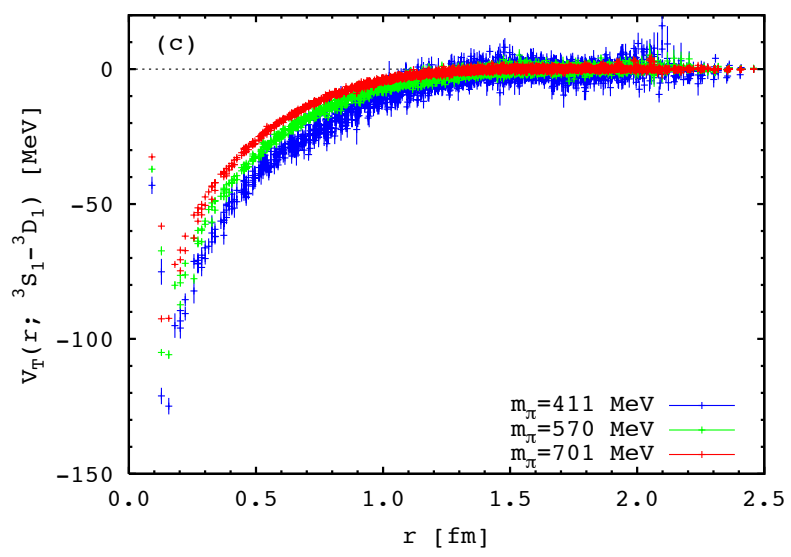

Fig. 6. Tensor potentials in $2+1$ flavor $\mathrm{QCD}$ as a function of $r$ at $m_{\pi} \simeq 701 \mathrm{MeV}$ (red), $570 \mathrm{MeV}$ (green) and $411 \mathrm{MeV}$ (blue). Taken from Ref. 37.

behavior of the potential, since lattice artifacts are large at short distances.

The meson theory predicts that the tensor potential receives a significant contribution from one-pion exchange, so that $V_{T}^{I=0}(r)$ is expected to be sensitive to the change of the pion mass. It is indeed the case, as shown in Fig. 6 A magnitude of $V_{T}^{I=0}(r)$ becomes larger as the pion mass decreases 37.

\subsection{Convergence of the velocity expansion}

In this subsection, we discuss the convergence of the velocity expansion in eq. (12). If the non-locality of the potential were absent, the LO potential would be exact at all energies (below the inelastic threshold). Therefore, we

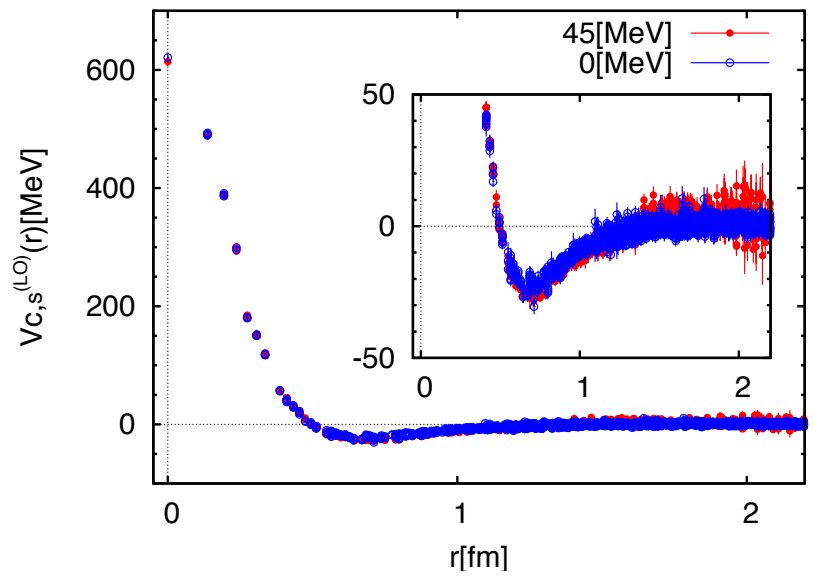

Fig. 7. The isospin-triplet (spin-singlet) central potential $V_{C}^{I=1}(r)$ obtained from the orbital $A_{1}^{+}$representation at $E_{k} \simeq$ $45 \mathrm{MeV}$ (red) and at $E \simeq 0$ (blue) in quenched QCD at $m_{\pi} \simeq 529 \mathrm{MeV}$. Taken from Ref. [38].

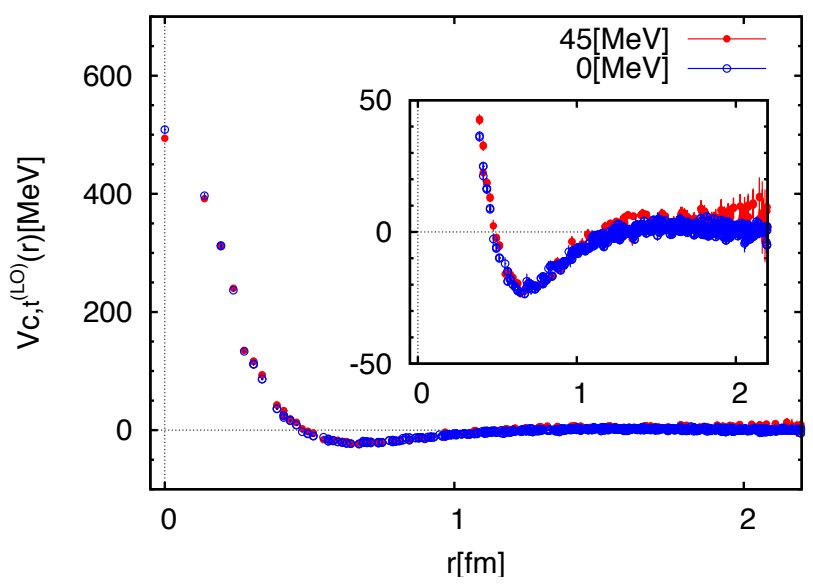

Fig. 8. The isospin-singlet (spin-triplet) central potential $V_{C}^{I=0}(r)$ obtained from the orbital $A_{1}^{+}-T_{2}^{+}$coupled channel at $E_{k} \simeq 45 \mathrm{MeV}$ (red) and at $E \simeq 0$ (blue) in quenched QCD at $m_{\pi} \simeq 529 \mathrm{MeV}$. Taken from Ref. 38.

can estimate a size of higher order terms in the velocity expansion, by considering the energy dependence of the local potential at the LO.

Potentials shown so far are extracted with the periodic boundary condition in spatial directions for quark fields, which leads to the almost zero kinetic energy $E_{k}$ for the ground state. With the anti-periodic boundary condition in spatial directions, on the other hand, we can significantly increase the ground state energy. To study the energy dependence of the LO potentials, we thus calculate the LO potentials at two different energies, $E_{k} \simeq 0$ $\mathrm{MeV}$ (periodic b.c.) and $45 \mathrm{MeV}$ (anti-periodic b.c.), in quenched $\mathrm{QCD}$ at $m_{\pi} \simeq 529 \mathrm{MeV}$ and $L \simeq 4.4 \mathrm{fm}$, by using the standard extraction of the potentials with the ground state saturation 38 . 


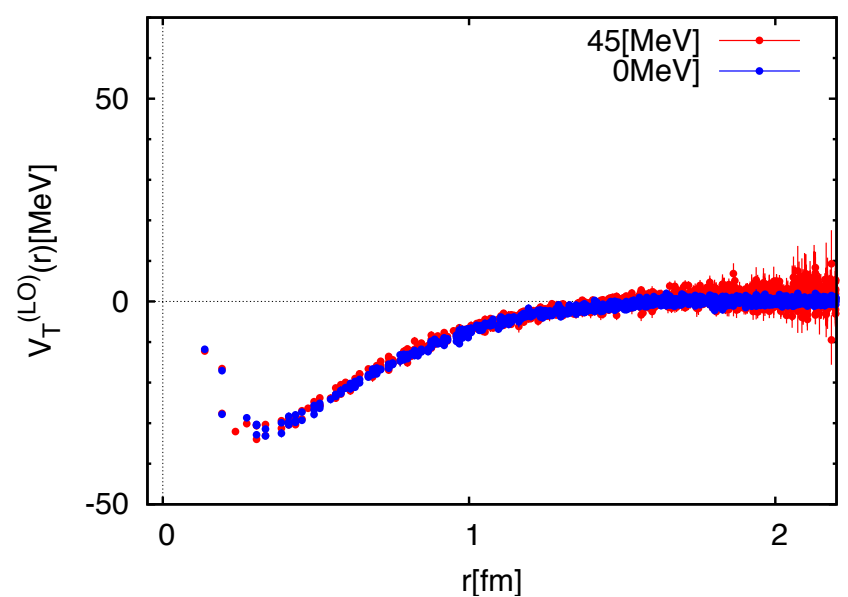

Fig. 9. The tensor potential $V_{T}^{I=0}(r)$ obtained from the orbital $A_{1}^{+}-T_{2}^{+}$coupled channel at $E_{k} \simeq 45 \mathrm{MeV}$ (red) and at $E \simeq$ 0 (blue) in quenched QCD at $m_{\pi} \simeq 529 \mathrm{MeV}$. Taken from Ref. 38.

With the anti-periodic boundary condition, 4 different momentum-wall sources, defined by

$$
q_{f}^{\text {wall }}\left(t_{0}\right) \equiv \sum_{\boldsymbol{x}} q\left(\boldsymbol{x}, t_{0}\right) f(\boldsymbol{x})
$$

are employed, where $f(\boldsymbol{x})=\cos (( \pm x \pm y+z) \pi / L)$, whereas $f(\boldsymbol{x})=1$ corresponds to the standard wall source used with the periodic boundary condition. The momentumwall sources generate the $L=T_{2}^{+}$state in addition to the $L=A_{1}^{+}$state.

Fig. 7 compares the isospin-triplet (spin-singlet) central potential $V_{C}^{I=1}(r)$ obtained from $A_{1}^{+}$state at $E_{k} \simeq 45$ $\mathrm{MeV}$ (red) with that at $E_{k} \simeq 0 \mathrm{MeV}$ (blue), while comparisons are made for the isospin-singlet (spin-triplet) central potential $V_{C}^{I=0}(r)$ and tensor potential $V_{T}^{I=0}(r)$ in Fig. 8 and Fig. 9. respectively 38. They are obtained from the orbital $A_{1}^{+}-T_{2}^{+}$coupled channel via eqs. (26) and (27). As seen from these figures, good agreements between two energies for all three cases indicate that higher order contributions in the velocity expansion are small in the energy region between $0 \mathrm{MeV}$ and $45 \mathrm{MeV}$. This means that the local potentials obtained at $E \simeq 0$ can be used to describe $N N$ scattering phase shifts in both isospin-triplet and singlet channels for the energy up to $45 \mathrm{MeV}$, at this pion mass in quenched QCD.

Higher order contributions in the velocity expansion of the non-local potential may also become manifest in the orbital angular momentum dependence of the local potential, since the orbital angular momentum $\boldsymbol{L}=\boldsymbol{r} \times \boldsymbol{p}$ contains one derivative. Fig. 10 compares the isospin-triplet (spin-singlet) potential $V_{C}^{I=1}(r)$ obtained from the $L=$ $A_{1}^{+}$state (red), whose main component has $L=0$, with the one from the $L=T_{2}^{+}$state (cyanogen), which mainly has the $L=2$ component 38 . In both cases, the local potential is determined at the same energy, $E_{k} \simeq 45, \mathrm{MeV}$, but at different orbital angular momentum. Although statistical errors are large for the $L=T_{2}^{+}$case, an agreement

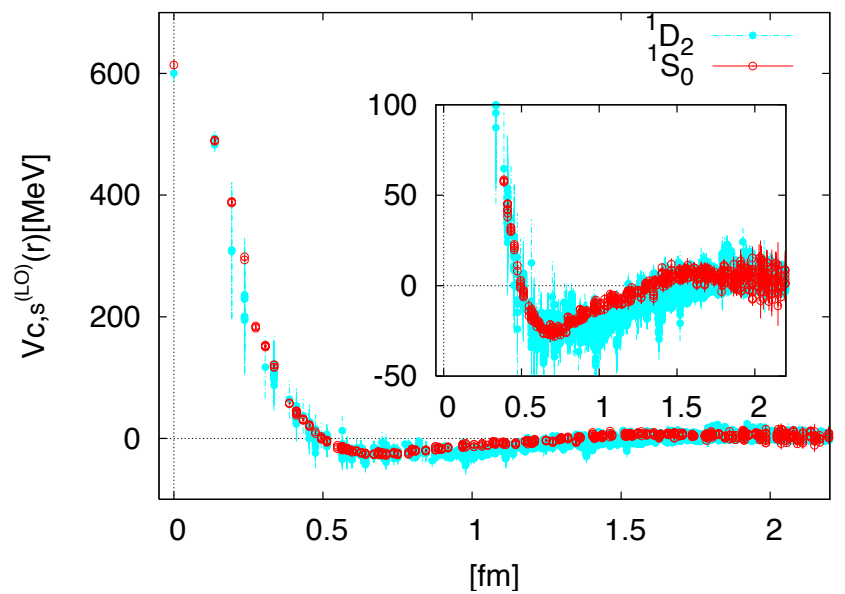

Fig. 10. The isospin-triplet (spin-singlet) central potential $V_{C}^{I=1}(r)$ at $E_{k} \simeq 45 \mathrm{MeV}$, obtained from the orbital $A_{1}^{+}$representation (red) and from the $T_{2}^{+}$representation (cyanogen), in quenched QCD at $m_{\pi} \simeq 529 \mathrm{MeV}$. Taken from Ref. 38.

between the two is observed, suggesting that the $L$ dependence is also small.

Comparisons in the above tell us that both energy and orbital angular momentum dependences for local LO potentials are almost invisible within statistical errors. We therefore conclude that contributions from higher order terms in the velocity expansion are small, so that these LO potential obtained at $E \simeq 0$ and $L=0$ are good approximations for the non-local potentials at least up to the energy $E_{k} \simeq 45 \mathrm{MeV}$ for both $L=0$ and 2 .

\section{Conclusions and discussions}

We review the HAL QCD method, recently proposed to extract hadronic interactions via lattice QCD simulations. We particularly focus on the methodology of this approach such as the strategy, the theoretical foundation and numerical techniques, of the HAL QCD method.

The equal-time NBS wave function for two nucleons plays a major role in the HAL QCD method, since it is proven that the NBS wave function encodes the $N N$ scattering phase shift in its asymptotic behavior at large separation. We therefore define the non-local potential, which can be constructed to be energy-independent, through the Schrödinger equation. By construction, this non-local but energy-independent potential gives the correct phase shift for the $N N$ scattering in QCD at all energies below the inelastic threshold. In practice, we expand the non-local potential in terms of velocities and truncate this velocity expansion at the lowest few orders. Once the (approximated) potential is obtained in the velocity expansion, we can calculate the $N N$ phase shift approximately at all energies below the inelastic threshold, by solving the Schrödinger equation with this potential.

In lattice QCD simulations, as in the case of the hadron mass extraction from a 2-pt correlation function, the NBS 
wave function can be extracted by the ground state saturation for the $N N$ 4-pt correlation functions, which however requires the large time separation and thus causes a large statistical fluctuation. To overcome this difficulty, we have proposed an improve method which directly gives the potential from the $N N$ 4-pt correlation functions without the ground state saturation. We have shown in $(2+1)$ flavor QCD simulations that the improved method works well to determine the isospin-triplet potential $V_{C}^{I=1}(r)$ at the LO of the velocity expansion, which reproduces a structure of the phenomenological potentials such as the repulsive core at short distance surrounded by the attractive well at medium and long distances. We have also shown the $N N$ scattering phase shift calculated with this potential as well as the isospin-singlet potentials $V_{C}^{I=0}(r)$ and $V_{T}^{I=0}(r)$ at the LO. We have estimated possible contributions from higher order terms in the velocity expansion, by studying the energy dependence as well as the $L$ dependence of the LO potentials in quenched QCD and have found that such contributions are rather small at low energy and small $L$.

The HAL QCD method is general and can be applied to other cases, which will be mentioned below with some references.

So far $N N$ potentials in our calculations are restricted to the parity even sector. We recently extend our study to the parity odd sector including the $\boldsymbol{L} \cdot \boldsymbol{S}$ potential, which appears at the next-to-leading order of the velocity expansion 39 . In the HAL QCD method, the three nuclear force can also be investigated and an indication of the short-distance repulsion is found in 2-flavor QCD simulations with the heavy pion mass 15, 16.

The HAL QCD method for the $N N$ potentials can be easily applied to potentials between other baryons. The $N \Xi$ potential was calculated in quenched QCD simulations, as the first attempt to study nucleon-hyperon interactions 7 , and soon after the $N \Lambda$ potential has been calculated in both quenched and full QCD simulations 8 . As more general cases, interactions between octet baryons are investigated in the flavor SU(3) limit, where up, down and strange quark masses are all degenerate 9, 11. Among 6 independent potentials corresponding to irreducible representations of the flavor $\mathrm{SU}(3)$ group, the flavor singlet potential is found to be attractive enough at all distances to have one bound state 10 , which corresponds to the H-dibaryon, predicted in Ref. 40. In order to analyze the property of the H-dibaryon in the real world where the strange quark is much heavier that the up and down quarks, the generalization of the HAL QCD method to the coupled channel potentials is required 41, 42, with which the baryon-baryon potentials in the $S=-2$ sector are investigated in (2+1)-flavor QCD simulations 43, 44, where $S$ represents the strangeness.

There are a few studies on meson-baryon interactions by the HAL QCD method such as $K N[12,13$ and charmonium and nucleon 14 .

Short distance behaviors of the potentials defined in the HAL QCD scheme can be investigated analytically by the operator product expansion and the renormalization group in the perturbative QCD, for the $N N$ 45, 46, the baryon-baryon [4], the $3 N$ [8], and the 3 baryons 49]. See Ref. 50] for a review on these results.

It is shown recently that the energy independent nonlocal coupled channel potentials exist even above the inelastic threshold 51. This result opens a possibility that the hadronic interaction can be extracted in lattice QCD at all energies without theoretical restrictions..

Finally, needless to say, the next step must be to calculate the $N N$ potential at physical pion mass. It is a great challenge for lattice QCD to show that the deuteron indeed bounds while di-neutron does not at the physical point.

\section{Acknowledgement}

I would like to thank all members of the HAL QCD collaboration for useful discussions during researches performed together. This work is supported in part by the Grants-inAid for Scientific Research on Innovative Areas (No.2004: 20105001, 20105003) and SPIRE (Strategic Program for Innovative Research).

\section{Appendix: Asymptotic behavior of the NBS wave function}

While the asymptotic behavior of the NBS wave function at large $r$ for the elastic $\pi \pi$ case has been shown in Refs. 52,53 and extended to the elastic $N N$ case in Ref. 21, we give a different derivation for it in this appendix, using the Lippmann-Schwinger equation (1). For simplicity, we consider the case of the scalar fields.

The unitarity of the S-matrix implies

$$
T^{\dagger}-T=i T^{\dagger} T
$$

which can be solved for the two particle scattering as

$$
\begin{aligned}
T \equiv \frac{1}{2 \pi} T\left(\boldsymbol{q}_{1}, \boldsymbol{q}_{2}\right) & =\frac{1}{2 \pi} \sum_{L, M} T_{L}(q, q) Y_{L M}\left(\Omega_{\boldsymbol{q}_{1}}\right) \overline{Y_{L M}\left(\Omega_{\boldsymbol{q}_{2}}\right)} \\
T_{L}(q, q) & =-\frac{8}{q E_{q}} e^{i \delta_{L}(q)} \sin \delta_{L}(q),
\end{aligned}
$$

where $q=\left|\boldsymbol{q}_{1}\right|=\left|\boldsymbol{q}_{2}\right|, Y_{L M}$ is the spherical harmonic function, $\Omega_{\boldsymbol{q}}$ is the solid angle of the vector $\boldsymbol{q}$, and $\delta_{L}(q)$ is the scattering phase shift for the partial wave with the angular momentum $L$ at energy $E_{q}=2 \sqrt{q^{2}+m^{2}}$ with the mass of the scalar particle $m$.

The equal-time NBS wave function for two scalar particle is defined by

$$
\begin{aligned}
\Psi_{\boldsymbol{k}}(\boldsymbol{x}) & ={ }_{\mathrm{in}}\left\langle 0\left|\varphi^{2}(\boldsymbol{x}, 0)\right| \boldsymbol{k}\right\rangle_{\text {in }}, \\
\varphi^{2}(\boldsymbol{x}, 0) & \equiv T\left\{\varphi_{1}(\boldsymbol{r}+\boldsymbol{x}, 0) \varphi_{2}(\boldsymbol{r}, 0)\right\}
\end{aligned}
$$

where, for simplicity, we assume that two scalar fields $\varphi_{1}$ and $\varphi_{2}$ have the same mass $m$. From the LippmannSchwinger equation (1), we have

$$
|0\rangle_{\text {in }}=|0\rangle_{0}+\int d \boldsymbol{q} \frac{|\boldsymbol{q}\rangle_{0} T_{\boldsymbol{q} 0}}{E_{0}-E_{q}+i \varepsilon}
$$


for the vacuum instate. The contribution of eq. (34) to the NBS wave function at large $r=|\boldsymbol{x}|$ amounts to

$$
{ }_{\text {in }}\left\langle 0\left|\varphi^{2}(\boldsymbol{x}, 0)\right| \boldsymbol{k}\right\rangle_{0} \simeq \frac{1}{Z_{k}}{ }_{0}\left\langle 0\left|\varphi^{2}(\boldsymbol{x}, 0)\right| \boldsymbol{k}\right\rangle_{0},
$$

where $Z_{k}$ is the normalization factor whose deviation from unity comes from the off-shell $T$-matrix $T_{\boldsymbol{q} 0}$. Using this, the NBS wave function becomes

$$
\begin{aligned}
\Psi_{\boldsymbol{k}}(\boldsymbol{x}) & =\frac{1}{Z_{k}} 0\left\langle 0\left|\varphi^{2}(\boldsymbol{x}, 0)\right| \boldsymbol{k}\right\rangle_{0} \\
& +\int d \boldsymbol{q} \frac{1}{Z_{q}} \frac{{ }_{0} \frac{\left\langle 0\left|\varphi^{2}(\boldsymbol{x}, 0)\right| \boldsymbol{q}\right\rangle_{0} T_{\boldsymbol{q} \boldsymbol{k}}}{E_{\boldsymbol{k}}-E_{\boldsymbol{q}}+i \varepsilon}}{}
\end{aligned}
$$

Inserting the expression that

$$
{ }_{0}\left\langle 0\left|\varphi^{2}(\boldsymbol{x}, 0)\right| \boldsymbol{k}\right\rangle_{0}=\frac{1}{(2 \pi)^{3} 2 E_{k}} e^{i \boldsymbol{k} \cdot \boldsymbol{x}},
$$

with $|\boldsymbol{k}\rangle_{0} \equiv a_{1}^{\dagger}(\boldsymbol{k}) a_{2}^{\dagger}(-\boldsymbol{k})|0\rangle_{0}$, into eq. 36, we have

$$
\begin{aligned}
\Psi_{\boldsymbol{k}}(\boldsymbol{x}) & =\frac{1}{2 E_{k} Z_{k}}\left[\frac{e^{i \boldsymbol{k} \cdot \boldsymbol{x}}}{(2 \pi)^{3}}\right. \\
& \left.+\int \frac{d^{3} q}{(2 \pi)^{3}} \frac{Z_{k} E_{k}}{Z_{q} E_{q}} \frac{e^{i \boldsymbol{q} \cdot \boldsymbol{x}} T(\boldsymbol{q}, \boldsymbol{k})}{4 \pi\left(E_{k}-E_{q}+i \epsilon\right)}\right] .
\end{aligned}
$$

With expressions that

$$
\begin{aligned}
e^{i \boldsymbol{k} \cdot \boldsymbol{x}} & =4 \pi \sum_{L, M} i^{L} j_{L}(k r) Y_{L M}\left(\Omega_{\boldsymbol{x}}\right) \overline{Y_{L M}\left(\Omega_{\boldsymbol{k}}\right)}, \\
\Psi_{\boldsymbol{k}}(\boldsymbol{x}) & =\sum_{L, M} i^{l} \Psi_{L}(r, k) Y_{L M}\left(\Omega_{\boldsymbol{x}}\right) \overline{Y_{L M}\left(\Omega_{\boldsymbol{k}}\right)}
\end{aligned}
$$

where $j_{L}(x)$ is the spherical Bessel function of the first kind, we obtain

$$
\begin{aligned}
\Psi_{L}(r, k) & =\frac{4 \pi}{(2 \pi)^{3} 2 E_{k} Z_{k}}\left[j_{L}(k r)\right. \\
& \left.+\int_{0}^{\infty} \frac{q^{2} d q}{2 \pi} \frac{Z_{k} E_{k}}{Z_{q} E_{q}} \frac{j_{L}(q r) T_{L}(q, k)}{2\left(E_{k}-E_{q}+i \varepsilon\right)}\right] .
\end{aligned}
$$

Under the assumption that $T_{L}(k, q)$ does not have any poles in the positive real axis for $E_{k}$ below the inelastic threshold, we perform the $q$ integral using the formula

$$
\int d q \frac{j_{L}(q r)}{k^{2}-q^{2}+i \varepsilon} F_{L}(q) \simeq-\frac{\pi}{2 k} F_{L}(k)\left[h_{L}(k r)+i j_{L}(k r)\right]
$$

for $r \gg 1$, where $F_{L}(q)$ does not have any poles in the positive real axis and satisfies $F_{L}(-q)=(-1)^{L} F_{L}(q)$, and $n_{L}(x)$ is the the spherical Bessel function of the second kind. After the $q$ integral, the second term in eq. 411 becomes

$$
\begin{aligned}
& -\left[n_{L}(k r)+i j_{L}(k r)\right] \frac{k E_{k}}{8} T_{L}(k, k) \\
& =\left[n_{L}(k r)+i j_{L}(k r)\right] \times e^{i \delta_{L}(k)} \sin \delta_{L}(k),
\end{aligned}
$$

where the unitarity constraint 32 for $T_{L}(k, k)$ is used. We then finally obtain

$$
\begin{aligned}
\Psi_{L}(r, k) & \simeq C_{L}(k)\left[j_{L}(k r) \cos \delta_{L}(k)+n_{L}(k r) \sin \delta_{L}(k)\right] \\
& \simeq C_{L}(k) \frac{\sin \left(k r-L \pi / 2+\delta_{L}(k)\right)}{k r}
\end{aligned}
$$

for $r \gg 1$, where asymptotic behaviors that $j_{L}(x) \simeq$ $\sin (x-L \pi / 2) / x$ and $n_{L}(x) \simeq \cos (x-L \pi / 2) / x$ are used, and the constant $C_{L}(k)$ is given by

$$
C_{L}(k)=\frac{4 \pi e^{i \delta_{L}(k)}}{(2 \pi)^{3} 2 E_{k} Z_{k}} .
$$

The phase appeared in the $T$-matrix,$\delta_{L}(k)$, can be interpreted as the scattering phase shift of the NBS wave function.

\section{References}

1. Z. Fodor and C. Hoelbling, Rev. Mod. Phys. 84 (2012) 449.

2. M. Lüscher, Nucl. Phys. B354 (1991) 531.

3. K. Orginos, PoS LATTICE2011 (2011) 016.

4. N. Ishii, S. Aoki and T. Hatsuda, Phys. Rev. Lett. 99 (200) 022001.

5. S. Aoki, T. Hatsuda and N. Ishii, Comput. Sci. Dis. 1 (2008) 015009.

6. S. Aoki, T. Hatsuda and N. Ishii, Prog. Theor. Phys. 123 (2010) 89

7. H. Nemura, N. Ishii, S. Aoki and T. Hatsuda, Phys. Lett. B673 (2009) 136.

8. H. Nemura, N. Ishii, S. Aoki and T. Hatsuda [PACS-CS Collaboration], PoS LATTICE2008 (2008) 156.

9. T. Inoue et al. [HAL QCD collaboration], Prog. Theor. Phys. 124 (2010) 591.

10. T. Inoue et al. [HAL QCD Collaboration], Phys. Rev. Lett. 106 (2011) 162002.

11. T. Inoue et al. [HAL QCD Collaboration], Nucl. Phys. A881 (2012) 28.

12. Y. Ikeda et al., PoS LATTICE2010 (2010) 143.

13. Y. Ikeda [HAL QCD Collaboration], PoS LATTICE 2011 (2011) 159 arXiv:1111.2663 [hep-lat]].

14. T. Kawanai and S. Sasaki, Phys. Rev. D82 (2010) 091501.

15. T. Doi for HAL QCD Collaboration, PoS LATTICE2010 (2010) 136.

16. T. Doi et al. [HAL QCD Collaboration], Prog. Theor. Phys. 127 (2012) 723.

17. S. Aoki for HAL QCD Collaboration, Prog. Part. Nucl. Phys. 66 (2011) 687.

18. S. Aoki et al. [HAL QCD Collaboration], Prog. Theor. Exp. Phys. 2012 (2012) 01A106.

19. S. Weinberg, The Quantum Theory of Fields, Volume I Foundations (Cambridge University Press, Cambridge, United Kingdom, 1999) p.155, Chapter 3.

20. J. Balog, M. Niedermaier, F. Niedermayer, A. Patrascioiu,

E. Seiler and P. Weisz, Nucl. Phys. B618 (2001) 315.

21. N. Ishizuka, PoS LAT2009 (2009) 119.

22. R. Tamagaki and W. Watari, Prog. Theor. Phys. Suppl. 39 (1967) 23.

23. S. Okubo and R. E. Marshak, Ann. Phys. 4 (1958) 166.

24. K. Nishijima, Phys. Rev. 111 (1958) 153. 
25. W. Zimmermann, Nuovo Cim. 10 (1958) 597.

26. R. Haag, Phys. Rev. 112 (1958) 669.

27. G. Parisi, Phys. Rept. 103 (1984) 203.

28. G. P. Lepage, in From Actions to Answers: Proceedings of the TASI 1989, edited by T. Degrand and D. Toussaint (World Scientific, Singapore, 1990).

29. N. Ishii et al. [HAL QCD Collaboration], Phys. Lett. B712 (2012) 437.

30. Y. Kuramashi, Prog. Theor. Phys. Suppl. 122 (1996) 153.

31. S. Aoki et al. [PACS-CS Collaboration], Phys. Rev. D79 (2009) 034503.

32. R. Machleidt, Phys. Rev. C63 (2001) 024001.

33. V. G. J. Stoks, R. A. M. Klomp, C. P. F. Terheggen and

J. J. de Swart, Phy. Rev. C49 (1994) 2950.

34. R. B. Wiringa, V. G. J. Stoks and R. Schiavilla, Phy. Rev. C51 (1995) 38.

35. In Research Highlights 2007, Nature 450 (2007) 1130.

36. http://www.nn-online.org/

37. N. Ishii [PACS-CS and HAL-QCD Collaborations], PoS LAT2009 (2009) 019

38. K. Murano, N. Ishii, S. Aoki and T. Hatsuda, Prog. Theor. Phys. 125 (2011) 1225.

39. K. Murano [HALQCD Collaboration], PoS LATTICE2011 (2011) 319.

40. R. L. Jaffe, Phys. Rev. Lett. 38 (1977) 195 [Erratum-ibid. 38 (1977) 617].

41. N. Ishii, for HAL QCD Collaboration, PoS LATTICE2010 (2010) 145.

42. S. Aoki et al. [HAL QCD Collaboration], Proc. Japan Acad. B87 (2011) 509.

43. K. Sasaki [HAL QCD Collaboration], PoS LATTICE2010 (2010) 157.

44. K. Sasaki [HAL QCD Collaboration], PoS LATTICE2011 (2011) 173.

45. S. Aoki, J. Balog and P. Weisz, PoS LATTICE 2009 (2009) 132.

46. S. Aoki, J. Balog and P. Weisz, JHEP 1005 (2010) 008.

47. S. Aoki, J. Balog and P. Weisz, JHEP 1009 (2010) 083.

48. S. Aoki, J. Balog and P. Weisz, New J. Phys. 14 (2012) 043046 .

49. S. Aoki, J. Balog and P. Weisz, Prog. Theor. Phys. 128 (2012) 1269

50. S. Aoki, J. Balog, T. Doi, T. Inoue and P. Weisz, Int. J. Mod. Phys. E 22 (2013) 1330012.

51. S. Aoki, B. Charron, T. Doi, T. Hatsuda, T. Inoue and N. Ishii, Phys. Rev. D 87 (2013) 034512.

52. C. J. D. Lin, G. Martinelli, C. T. Sachrajda and M. Testa, Nucl. Phys. B619 (2001) 467.

53. S. Aoki et al. [CP-PACS Collaboration], Phys. Rev. D71 (2005) 094504. 